\title{
Long-Term Effects of Intensive Glucose Lowering on Cardiovascular Outcomes
}

\author{
The ACCORD Study Group ${ }^{\star}$
}

\begin{abstract}
BACKGROUND—Intensive glucose lowering has previously been shown to increase mortality among persons with advanced type 2 diabetes and a high risk of cardiovascular disease. This report describes the 5-year outcomes of a mean of 3.7 years of intensive glucose lowering on mortality and key cardiovascular events.
\end{abstract}

\begin{abstract}
METHODS-We randomly assigned participants with type 2 diabetes and cardiovascular disease or additional cardiovascular risk factors to receive intensive therapy (targeting a glycated hemoglobin level below 6.0\%) or standard therapy (targeting a level of 7 to $7.9 \%$ ). After termination of the intensive therapy, due to higher mortality in the intensive-therapy group, the target glycated hemoglobin level was 7 to $7.9 \%$ for all participants, who were followed until the planned end of the trial.
\end{abstract}

RESULTS-Before the intensive therapy was terminated, the intensive-therapy group did not differ significantly from the standard-therapy group in the rate of the primary outcome (a composite of nonfatal myocardial infarction, nonfatal stroke, or death from cardiovascular causes) $(\mathrm{P}=0.13$ ) but had more deaths from any cause (primarily cardiovascular) (hazard ratio, 1.21; 95\% confidence interval [CI], 1.02 to 1.44) and fewer nonfatal myocardial infarctions (hazard ratio, $0.79 ; 95 \% \mathrm{CI}, 0.66$ to 0.95 ). These trends persisted during the entire follow-up period (hazard ratio for death, $1.19 ; 95 \%$ CI, 1.03 to 1.38 ; and hazard ratio for nonfatal myocardial infarction, 0.82 ; $95 \%$ CI, 0.70 to 0.96 ). After the intensive intervention was terminated, the median glycated hemoglobin level in the intensive-therapy group rose from $6.4 \%$ to $7.2 \%$, and the use of glucoselowering medications and rates of severe hypoglycemia and other adverse events were similar in the two groups.

CONCLUSIONS-As compared with standard therapy, the use of intensive therapy for 3.7 years to target a glycated hemoglobin level below $6 \%$ reduced 5-year nonfatal myocardial infarctions but increased 5-year mortality. Such a strategy cannot be recommended for high-risk patients with advanced type 2 diabetes. (Funded by the National Heart, Lung and Blood Institute;

ClinicalTrials.gov number, NCT00000620.)

Copyright $\odot 2011$ Massachusetts Medical Society.

Address reprint requests to Dr. Gerstein at McMaster University, Department of Medicine, HSC 3V38, 1200 Main St. W., Hamilton, ON L8N 3Z5, Canada, or at gerstein @ mcmaster.ca

* Members of the Action to Control Cardiovascular Risk in Diabetes (ACCORD) Study Group are listed in the Supplementary Appendix, available at NEJM.org.

No other potential conflict of interest relevant to this article was reported.

Disclosure forms provided by the authors are available with the full text of this article at NEJM.org. 
Type 2 diabetes mellitus is a strong, independent risk factor for cardiovascular disease and death, ${ }^{1}$ and many epidemiologic analyses have identified a progressive relationship between hyperglycemia and these outcomes. ${ }^{2-5}$ The Action to Control Cardiovascular Risk in Diabetes (ACCORD) trial was designed to determine whether a strategy of targeting normal glycated hemoglobin levels (i.e., $<6.0 \%$ ) would reduce the risk of serious cardiovascular events in middle-aged and elderly people with type 2 diabetes mellitus, glycated hemoglobin levels of $7.5 \%$ or more, and additional cardiovascular risk factors. ${ }^{6}$ However, on the basis of a mean of 3.5 years' worth of data, the independent data and safety monitoring board recommended termination of the intensive glucose-lowering regimen because of the finding of higher mortality in the intensive-therapy group. Therefore, we applied the approaches that were used in the standard control group to participants assigned to the intensive-therapy group, for up to 17 months of additional follow-up. We report the clinical outcomes at 5 years of follow-up in response to a mean of 3.7 years of an intensive glycemia strategy.

\section{METHODS}

\section{STUDY DESIGN}

The design and major results of the trial have been published previously. ${ }^{6,7}$ Briefly, we recruited male and female volunteers from 77 clinical centers in the United States and Canada. The participants were 40 to 79 years of age, had type 2 diabetes mellitus and a glycated hemoglobin level of $7.5 \%$ or more, and had previous evidence of cardiovascular disease or risk factors for cardiovascular disease. Participants were randomly assigned to receive either intensive glucose-lowering therapy targeting a glycated hemoglobin level of less than $6.0 \%$ or standard glucose-lowering therapy targeting a level of 7 to $7.9 \%$. All participants received counseling about lifestyle and education about the management of diabetes. Glucose-lowering drugs were chosen from a common formulary according to the participant's studygroup assignment and response to therapy. ${ }^{7}$ Glycated hemoglobin levels were audited regularly according to treatment group and study center, and feedback was provided to facilitate the attainment of the target glycated hemoglobin levels.

\section{TERMINATION OF INTENSIVE REGIMEN AND ASSESSMENT OF OUTCOMES}

Recruitment occurred in two phases, from January to June 2001 and from February 2003 to October 2005. On February 5, 2008, participants were informed of the decision to discontinue the intensive glucose-lowering regimen, after a mean treatment period of 3.7 years. Participants in the intensive-therapy group subsequently were switched to standard glycemic therapy, and their target glycated hemoglobin level of less than $6 \%$ was changed to a target level of 7 to $7.9 \%$. Since participants had also been assigned to receive treatment either to control lipid levels or to lower blood pressure, ${ }^{8,9}$ they continued to be followed at least every 4 months until the originally planned end of the trial (June 2009). Thus, data on clinical outcomes, including the primary outcome (a composite of nonfatal myocardial infarction, nonfatal stroke, or death from cardiovascular causes) and death from any cause (a secondary outcome), continued to be collected and adjudicated for an additional 17 months by a central committee whose members were unaware of study-group assignments. 


\section{INTERVENTION EFFECTS}

The effects of the glycemic intervention during a mean of 3.5 years (until December 10, 2007), which provided the basis for the data and safety monitoring board's recommendation to discontinue the intensive regimen, have been reported previously. ${ }^{6}$ Here we report the effect of the intervention during an additional 0.2 years (i.e., until February 5, 2008), which was when participants were informed of the change in approach. We also report on outcomes that occurred before February 5, 2008, that were not reported to the coordinating center as of December 10, 2007. Therefore, we used intention-to-treat analyses to report on the effect of a mean of 3.7 years of an intensive glycemic intervention on cardiovascular disease, followed by a mean of 1.2 years of standard glycemic therapy. Also reported are the effects of the glycemic intervention until the transition date and until the end of the overall trial for both the blood-pressure and lipid trials. The treatment effects of the lipid and bloodpressure interventions were reported separately. ${ }^{8,9}$ All primary and secondary outcomes were adjudicated centrally by two adjudicators who were unaware of treatment-group assignments, and in addition, deaths were reviewed by two diabetes experts (who were unaware of treatment-group assignments) to determine whether they were due to hypoglycemia. The ACCORD trial was sponsored by the National Heart, Lung, and Blood Institute (NHLBI), and the protocol (available with the full text of this article at NEJM.org) was approved by an NHLBI review panel and by the ethics committee at each center. All participants provided written informed consent. All authors vouch for the accuracy and completeness of the reported data. The donors of medications and devices had no role in the study design, data accrual and analysis, or manuscript preparation.

\section{STATISTICAL ANALYSIS}

All statistical analyses were conducted at the coordinating center with the use of S-Plus software, version 8.0 (Insightful), or SAS software, version 9.2 (SAS Institute). Baseline characteristics of the participants were summarized with the use of means, standard deviations, and percentages. Median glycated hemoglobin levels were calculated monthly (by calendar month) to show the effect of the switch from intensive therapy to the standard approach. Exposure to glucose-lowering drugs was summarized as the number of participants who were prescribed a medication at the last visit before the transition date and at the trial termination. The incidence of key safety outcomes was expressed as the percentage of events per follow-up year, taking into account censoring of follow-up data. Kaplan-Meier estimates were used to calculate the percentage of participants who had an event during follow-up.

Primary and secondary outcomes were analyzed with the use of Cox proportional-hazards regression analyses according to the intention-to-treat principle, and between-group comparisons of the outcomes were performed with the use of hazard ratios and $95 \%$ confidence intervals derived from these models. These analyses were performed for events occurring from randomization until the date of transition (February 5, 2008) and from randomization until the final visit (between the beginning of March and the end of June 2009). An additional post hoc analysis was performed for the primary outcome and death from any cause with the use of data from the post-transition phase only. 
For analyses of outcomes, data from participants without final follow-up data were censored as of the time of their last completed 4-month visit in both the intensive-therapy and standard-therapy groups. Data on mortality for participants in the United States who were not followed for the full follow-up period and who were not known to be deceased were censored as of the most recent date they were known to be alive or January 1, 2008, on the basis of the National Death Index.

Silent myocardial infarctions were identified on the basis of electrocardiograms obtained every 2 years and were considered to have occurred at the midpoint of the dates between the electrocardiogram showing a new myocardial infarction and the previous electrocardiogram. Information from electrocardiograms obtained after the transition date were not known to the data and safety monitoring board or investigators at the time of the transition. Therefore, new silent myocardial infarctions detected after the transition date that would have been assigned to the period before transition were deemed to have occurred on the date of transition; this occurred for 29 participants.

Cox models for the primary outcome contained a term representing study-group assignments plus terms accounting for the following prespecified stratifying variables: assignment to the blood-pressure trial or lipid trial; assignment to the intensive blood-pressure intervention in the blood-pressure trial; assignment to receive fibrate in the lipid trial; the seven clinical center networks; and the presence or absence of previous cardiovascular disease. For all secondary outcomes, an a priori decision was made to drop the clinical center networks from this model, because fewer events were expected than for the primary outcomes. The consistency of the effect of the study-group assignment on death from any cause and on the primary outcome in the blood-pressure trial and the lipid trial was assessed with the use of statistical tests of interactions between the treatment effect and the subgroup within the Cox models.

Unless otherwise indicated, nominal $\mathrm{P}$ values, unadjusted for the multiple tests performed for this report or for monitoring by the data and safety monitoring board, are reported. Since we conducted 46 statistical tests of hypotheses related to secondary end points and subgroups, there was a $91 \%$ chance (i.e., $1-[1-0.05]^{46}$ ) that at least one of these tests would be significant at an alpha level of 0.05 , assuming independence between tests.

The effect of the study-group assignment on the primary outcome or mortality after the transition in participants who had not had a primary outcome and who were alive at the transition date was explored with the use of Kaplan-Meier curves and Cox regression models. Further post hoc exploratory analyses to identify factors associated with higher mortality in the intensive-therapy group have examined baseline characteristics, ${ }^{10}$ the achieved glycated hemoglobin level and the rapidity of its decline, ${ }^{11}$ and hypoglycemic events. ${ }^{12,13}$

\section{RESULTS}

Baseline characteristics of the participants have been reported previously. ${ }^{6}$ Table 1 shows these characteristics before the transition date and at the final visit. Figure 1 in the 
Supplementary Appendix (available at NEJM.org) shows the completeness of follow-up in the two study groups. Median glycated hemoglobin levels before the transition date in the intensive-therapy and standard-therapy groups were $6.4 \%$ and $7.5 \%$, respectively. After the transition date, therapy was relaxed (i.e., fewer drugs or lower doses were used) for a particular indication at least as often in the intensive-therapy group as in the standardtherapy group. For example, at the first post-transition visit, relaxation of therapy was indicated in $94 \%$ of participants in the intensive-therapy group and $69 \%$ of those in the standard-therapy group. At the final visit, median glycated hemoglobin levels were $7.2 \%$ in the intensive-therapy group and 7.6\% in the standard-therapy group (Fig. 2 in the Supplementary Appendix). By the final visit, the numbers of participants who were receiving metformin, secretagogues, thiazolidinediones, insulin, and combination therapy with insulin and oral agents were similar in the two groups (Table 1 in the Supplementary Appendix). Rates of severe hypoglycemia and other adverse events within the two groups were similar after the transition (Table 2 in the Supplementary Appendix).

Figure 1 shows the incidence of the primary outcome and death from any cause from randomization until the time of transition, from randomization until the end of the whole study, and from the transition date until the termination of the trial. Figure 2 shows the effect of intensive glucose-lowering therapy on all the major outcomes, from randomization until the end of the active treatment period and until the end of the study. Before the transition, the incidence of the primary outcome among the participants in the intensive-therapy group was $2.0 \%$ per year, as compared with an incidence of $2.2 \%$ per year among the participants in the standard-therapy group (hazard ratio, $0.90 ; 95 \%$ confidence interval [CI], 0.78 to 1.03; nominal $\mathrm{P}=0.132$, and $\mathrm{P}=0.134$ after adjustment for repeat testing by the data and safety monitoring board) and remained nonsignificant throughout the entire period of observation (hazard ratio, $0.91 ; 95 \% \mathrm{CI}, 0.81$ to $1.03 ; \mathrm{P}=0.12$ ).

The intensive therapy had different effects on two of the key components of this primary outcome. At the time of the transition, the rate of nonfatal myocardial infarction in the intensive-therapy group was lower than that in the standard-therapy group $(1.08 \%$ vs. $1.35 \%$; hazard ratio, $0.79 ; 95 \% \mathrm{CI}, 0.66$ to $0.95 ; \mathrm{P}=0.01$ ), but the rate of death from cardiovascular causes was non-significantly higher $(0.71 \%$ vs. $0.55 \%$; hazard ratio, $1.27 \%$; $95 \%$ CI, 0.99 to $1.63 ; \mathrm{P}=0.07)$. These divergent effects were retained at the end of the study, with a rate of nonfatal myocardial infarction in the intensive-therapy group that was lower than that in the standard-therapy group (1.18 vs. 1.42 ; hazard ratio, $0.82 ; 95 \% \mathrm{CI}, 0.70$ to $0.96 ; \mathrm{P}=0.01)$ and a rate of death from cardiovascular causes that was higher $(0.74$ vs. 0.57 ; hazard ratio, $1.29 ; 95 \% \mathrm{CI}, 1.04$ to $1.60 ; \mathrm{P}=0.02$ ).

Finally, at the time of the transition, there was a $21 \%$ higher rate of death from any cause in the intensive-therapy group than in the standard-therapy group (1.42 vs. 1.16 ; $95 \% \mathrm{CI}, 1.02$ to 1.44 ; nominal $\mathrm{P}=0.030$ and $\mathrm{P}=0.036$ after adjustment for repeat testing by the data and safety monitoring board) and a $19 \%$ higher rate at the end of the study (1.53 vs. $1.27 ; 95 \%$ CI, 1.03 to 1.38; $\mathrm{P}=0.02$ ) (Fig. 1 and 2). The causes of death are listed in Table 2. There was no clear difference between study groups in any other predefined cardiovascular outcomes. 
Table 3 lists the annual incidence of the primary and secondary outcomes in the two treatment groups after the transition date, and Figures $1 \mathrm{C}$ and $1 \mathrm{~F}$ show the corresponding Kaplan-Meier curves for the primary outcome and death from any cause. Hazard ratios in the post-transition period were not significantly different from those in the pretransition period for either the primary outcome (ratio of pretransition to post-transition hazard ratios, $0.95 ; 95 \% \mathrm{CI}, 0.72$ to $1.26 ; \mathrm{P}=0.72$ ) or death from any cause (ratio of pretransition to posttransition hazard ratios, $1.06 ; 95 \% \mathrm{CI}, 0.76$ to $1.46 ; \mathrm{P}=0.74)$. There was a possible difference in the effect of the intensive therapy on the pretransition primary outcome among participants with a baseline glycated hemoglobin level of $8 \%$ or less as compared with those with a level of more than $8 \%(\mathrm{P}=0.03$ for interaction) (Fig. 3 in the Supplementary Appendix).

A total of 4733 participants were randomly assigned to receive either intensive or standard therapy to lower their blood pressure, and 5518 participants were randomly assigned to a statin plus either fenofibrate or placebo for control of low-density lipoprotein cholesterol. No significant interactions were noted between the glucose-lowering study and the bloodpressure study for the primary outcome, or between the glucose-lowering study and the lipid study for either the primary outcome or death from any cause. However, there was evidence of an interaction between the intensive glucose-lowering group and the intensive bloodpressure-lowering group with respect to death from any cause both before the transition $(\mathrm{P}=$ 0.03 for interaction) and at the end of the trial ( $\mathrm{P}=0.05$ for interaction) (Fig. 4 in the Supplementary Appendix). Before the transition, this interaction was characterized by a marginally higher mortality rate in the intensive glucose-lowering group than in the standard glucose-lowering group among participants also assigned to the intensive blood-pressurelowering group (hazard ratio, $1.45 ; 95 \% \mathrm{CI}, 1.00$ to $2.12 ; \mathrm{P}=0.05$ ) but not among those also assigned to the standard blood-pressure-lowering group (hazard ratio, $0.78 ; 95 \% \mathrm{CI}, 0.52$ to $1.18 ; \mathrm{P}=0.24)$.

\section{DISCUSSION}

The ACCORD trial involved persons who had had diabetes for a median of 10 years, with a glycated hemoglobin level of at least $7.5 \%$, and who had a high risk of cardiovascular disease. Our findings indicate that in a high-risk population such as this, a mean of 3.7 years of intensive therapy consisting of multiple glucose-lowering methods to target normal glycated hemoglobin levels (i.e., below 6.0\%) does not result in a significantly lower number of major cardiovascular events after 5 years than does an approach that uses similar methods to target levels that are more typically achieved in persons in the United States and Canada (i.e., 7 to $7.9 \%$ ). Indeed, the intensive approach led to more deaths. Effects on the primary outcome were similar during the 3.7-year glucose-lowering period and the entire 5year follow-up period; effects on mortality also were similar during the two periods. Similar effects on the primary outcome and mortality were noted in most of the predefined subgroups. The nominally positive tests for interaction with respect to the primary outcome and baseline glycated hemoglobin levels and with respect to death from any cause and the blood-pressure intervention may well have been due to chance, since a large number of statistical tests were performed. No inferences can be made about the effect of the intervention during the post-transition period, because between-group differences during this 
period alone are likely to have been driven by between-group differences in the characteristics of participants who survived and were followed during this period.

Reasons for the higher mortality in the intensive-therapy group during the pretransition period remain unclear. Because of the equivalent rates of hypoglycemia in the posttransition period, severe hypoglycemia cannot be implicated. Additional analyses reported elsewhere $^{12}$ also do not implicate severe hypoglycemia. According to other analyses, the degree of reduction in glycated hemoglobin levels cannot be implicated. ${ }^{11}$ Further analyses should explore possible explanations, such as the role of various drugs, drug combinations, or drug interactions; weight gain; the relatively short intervention period (3.7 years); and the observed interaction between the blood-pressure and glycemia trials with respect to mortality.

Strengths of our study include the randomized trial design, large sample, wide variety of clinics, frequent follow-up, high rate of complete follow-up, high rate of adherence to the study assignment, and adjudication of all events by a central committee that was unaware of the study-group assignments. The clinical relevance of the results is highlighted by the following facts: the approach used commonly available drugs, glycemia was managed within the context of good control of blood pressure and lipid levels, the recruited participants were representative of many people with diabetes who are currently receiving care in ambulatory settings, and several organizations have recommended glycemic targets of $6.5 \%$ or lower.

These findings are most applicable to middle-aged and older patients with a long duration of diabetes, a high risk of cardiovascular disease, and hyperglycemia and should be interpreted in light of the specific features of the ACCORD trial. For example, the ACCORD trial excluded people with glycated hemoglobin levels below 7.5\%. Moderate heterogeneity with respect to subgroups predefined by the glycated hemoglobin level at baseline (Fig. 3 in the Supplementary Appendix) suggests that participants whose glycated hemoglobin level at baseline was $8 \%$ or lower may have had a better response to therapy than participants with higher glycated hemoglobin levels. Although this hypothesis is clearly not proved by the ACCORD trial, it is supported by a recent epidemiologic analysis of the cardiovascular effect of glucose lowering in a cohort of people with type 2 diabetes. ${ }^{14}$

The ACCORD trial explicitly tested whether targeting a glycated hemoglobin level below $6 \%$ by means of a large menu of glucose-lowering agents is superior to targeting a glycated hemoglobin level of 7 to $7.9 \%$. Therefore, our findings should be interpreted in relation to these therapies and target glycated hemoglobin levels. Furthermore, targeting normal glycated hemoglobin levels (i.e., $<6.0 \%$ ) required the use of multiple combinations of glucose-lowering medications in ways that are not used in standard care. For example, $42 \%$ of participants in the intensive-therapy group were receiving three or more classes of oral agents, either alone (17\%) or in combination with insulin (25\%), whereas such combinations were used in $19 \%$ of the participants in the standard-therapy group (Table 1 in the Supplementary Appendix). Whether these unconventional combinations were responsible for the results and whether similar findings would have been observed with newer glucose- 
lowering therapies, different drug combinations, or different target glycated hemoglobin levels is unknown.

Finally, people with newly diagnosed diabetes may have a different response to intensive glucose-lowering therapy. A large trial involving people with newly diagnosed type 2 diabetes, in which normal glucose levels were targeted and a median glycated hemoglobin level of 7\% (as opposed to 7.9\%) was achieved, showed a neutral cardiovascular effect after 10 years but a reduced rate of myocardial infarction and death after 20 years. ${ }^{15}$

In summary, the results of the ACCORD trial show that in persons who have a high risk of cardiovascular disease and suboptimally controlled, long-standing diabetes, with good blood-pressure and lipid control, an intensive therapeutic approach targeting normal glycated hemoglobin levels with the use of multiple medications is associated with higher mortality than is a standard approach targeting higher glycated hemoglobin levels. The higher risk of death from any cause and from cardiovascular causes in the intensive-therapy group means that a therapeutic approach that targets glycated hemoglobin levels below $6 \%$ cannot be generally recommended in this population. Thus, the results of the ACCORD trial suggest a lower limit for glycemic targets, achieved with the use of multiple combinations of currently available approaches.

\section{Supplementary Material}

Refer to Web version on PubMed Central for supplementary material.

\section{Acknowledgments}

Supported by the National Heart, Lung, and Blood Institute (contracts N01-HC-95178, N01-HC-95179, N01HC-95180, N01-HC-95181, N01-HC-95182, N01-HC-95183, N01-HC-95184, IAA\#Y1-HC-9035, and IAA\#Y1HC-1010), and partially supported by the National Institute of Diabetes and Digestive and Kidney Diseases, the National Institute on Aging, and the National Eye Institute and by General Clinical Research Centers at many sites; substudies within the ACCORD trial on cost-effectiveness and health-related quality of life were supported by the Centers for Disease Control and Prevention. The following companies provided study medications, equipment, or supplies: Abbott Laboratories, Amylin Pharmaceutical, AstraZeneca, Bayer HealthCare, Closer Healthcare, GlaxoSmithKline, King Pharmaceuticals, Merck, Novartis, Novo Nordisk, Omron Healthcare, Sanofi-Aventis, and Schering-Plough.

Dr. Bigger reports receiving consulting fees and travel support from Merck and Roche and patent fees and royalties from the Massachusetts Institute of Technology for risk-stratification software; Dr. Buse, receiving consulting fees from Novo Nordisk, Amylin, Becton Dickinson, Eli Lilly, Hoffmann-La Roche (now Genentech), Glyco-Mark, Wyeth, Daiichi Sankyo, Bristol-Myers Squibb, Bayhill Therapeutics, LipoScience, MannKind, Veritas, MicroIslet, GlaxoSmithKline, Abbott, Exsulin, and GI Dynamics and grant support from Amylin, Novo Nordisk, Medtronic, Eli Lilly, Novartis, Tolerex, Osiris, Halozyme, Pfizer, Hoffmann-La Roche, InterKrin, Merck, Sanofi-Aventis, Dexcom, Johnson \& Johnson, Bristol-Myers Squibb, Fujisawa, and the American Academy of Family Practice Foundation, holding stock in Insulet, and providing expert testimony for Novo Nordisk; Dr. Cushman, receiving consulting fees from Novartis, Takeda, Sanofi-Aventis, Bristol-Myers Squibb, King, Daiichi-Sankyo, Gilead, Theravance, Pharmacopeia, and Sciele and institutional grant support to the Memphis Veterans Affairs Medical Center from Novartis, Glaxo-SmithKline, and Merck; Dr. Genuth, receiving consulting fees from Merck and Daiichi Sankyo and holding stock in Novartis and Johnson \& Johnson; Dr. Gerstein, receiving consulting fees from Sanofi-Aventis, GlaxoSmithKline, Eli Lilly, Novo Nordisk, AstraZeneca, Bristol-Myers Squibb, Roche, Medtronic, Merck, Bayer, Bioavail, and Janssen-Ortho, institutional grant support to McMaster University from SanofiAventis, GlaxoSmithKline, Novo Nordisk, Merck, Pronova, Roche, Eli Lilly, and Boehringer Ingelheim, and lecture fees from Sanofi-Aventis, GlaxoSmith-Kline, Solvay, Boehringer Ingelheim, Servier, Bayer, Eli Lilly, Novo Nordisk, and Takeda; Dr. Ginsberg, being a member of the board of Merck and Schering-Plough and the global advisory board of Bristol-Myers Squibb/AstraZeneca and receiving consulting fees from GlaxoSmithKline, Merck, Bristol-Myers Squibb, AstraZeneca, Regeneron/Sanofi-Aventis, Abbott, Roche, Isis/Genzyme, Novartis, and Pfizer, institutional grant support to the Columbia University College of Physicians and Surgeons from Merck, 
Roche, Isis/Genzyme, and AstraZeneca, and payment from Pfizer for development of an educational presentation; Dr. Goff, being a member of the data and safety monitoring board for Takeda and receiving institutional grant support to the Wake Forest University School of Medicine from Merck; Dr. Grimm, being a member of the board of Pfizer, receiving consulting fees from Pfizer, Merck, and Novartis, personal and institutional grants to the University of Minnesota from Pfizer, Merck, and Novartis, lecture fees from Pfizer, Merck, Novartis, Forest, Schering-Plough, and Takeda, and travel support from Takeda and Roche, and attending the AstraZeneca symposium at the Cleveland Clinic and investigator meetings for Merck, Novartis, and Pfizer; Dr. Ismail-Beigi, receiving consulting fees from Eli Lilly; Dr. Miller, receiving consulting fees from Roche; and Dr. Probstfield, receiving institutional grant support to the University of Washington School of Medicine from Sanofi-Aventis, Boehringer Ingelheim, and Abbott.

\section{Appendix}

The members of the writing group (Hertzel C. Gerstein, M.D., McMaster University and Hamilton Health Sciences, Hamilton, ON, Canada; Michael E. Miller, Ph.D., Wake Forest University School of Medicine, Winston-Salem, NC; Saul Genuth, M.D., Case Western Reserve University, Cleveland; Faramarz Ismail-Beigi, M.D., Ph.D., Case Western Reserve University, Cleveland; John B. Buse, M.D., Ph.D., University of North Carolina, Chapel Hill; David C. Goff, Jr., M.D., Ph.D., Wake Forest University School of Medicine, WinstonSalem, NC; Jeffrey L. Probstfield, M.D., University of Washington, Seattle; William C. Cushman, M.D., Memphis Veterans Affairs Medical Center, Memphis; Henry N. Ginsberg, M.D., Columbia University College of Physicians and Surgeons, New York; J. Thomas Bigger, M.D., Columbia University College of Physicians and Surgeons, New York; Richard H. Grimm, Jr., M.D., Ph.D, University of Minnesota, Berman Center for Outcomes and Clinical Research, Minneapolis; Robert P. Byington, Ph.D., Wake Forest University School of Medicine, Winston-Salem, NC; Yves D. Rosenberg, M.D., National Heart, Lung, and Blood Institute, Bethesda, MD; and William T. Friedewald, M.D., Columbia University College of Physicians and Surgeons, New York) assume responsibility for the content of this article.

\section{REFERENCES}

1. Huxley R, Barzi F, Woodward M. Excess risk of fatal coronary heart disease associated with diabetes in men and women: meta-analysis of 37 prospective cohort studies. BMJ. 2006; 332:73-78. [PubMed: 16371403]

2. Selvin E, Coresh J, Golden SH, Brancati FL, Folsom AR, Steffes MW. Glycemic control and coronary heart disease risk in persons with and without diabetes: the Atherosclerosis Risk in Communities study. Arch Intern Med. 2005; 165:1910-1916. [PubMed: 16157837]

3. Levitan EB, Liu S, Stampfer MJ, et al. HbA1C measured in stored erythrocytes and mortality rate among middle-aged and older women. Diabetologia. 2008; 51:267-275. [PubMed: 18043905]

4. Selvin E, Steffes MW, Zhu H, et al. Glycated hemoglobin, diabetes, and cardiovascular risk in nondiabetic adults. N Engl J Med. 2010; 362:800-811. [PubMed: 20200384]

5. Gerstein HC, Swedberg K, Carlsson J, et al. The hemoglobin A1c level as a progressive risk factor for cardiovascular death, hospitalization for heart failure, or death in patients with chronic heart failure: an analysis of the Candesartan in Heart failure: Assessment of Reduction in Mortality and Morbidity (CHARM) program. Arch Intern Med. 2008; 168:1699-1704. [PubMed: 18695086]

6. The Action to Control Cardiovascular Risk in Diabetes Study Group. Effects of intensive glucose lowering in type 2 diabetes. N Engl J Med. 2008; 358:2545-2559. [PubMed: 18539917]

7. Gerstein HC, Riddle MC, Kendall DM, et al. Glycemia treatment strategies in the Action to Control Cardiovascular Risk in Diabetes (ACCORD) trial. Am J Cardiol. 2007; 99(12A):34i-43i.

8. The ACCORD Study Group. Effects of combination lipid therapy in type 2 diabetes mellitus. N Engl J Med. 2010; 362:1563-1574. [Erratum, N Engl J Med 2010;362:1748.]. [PubMed: 20228404] 
9. Idem. Effects of intensive blood-pressure control in type 2 diabetes mellitus. N Engl J Med. 2010; 362:1575-1585. [PubMed: 20228401]

10. Calles-Escandón J, Lovato LC, Simons-Morton DG, et al. Effect of intensive compared with standard glycemia treatment strategies on mortality by baseline subgroup characteristics: the Action to Control Cardiovascular Risk in Diabetes (ACCORD) trial. Diabetes Care. 2010; 33:721727. [PubMed: 20103550]

11. Riddle MC, Ambrosius WT, Brillon DJ, et al. Epidemiologic relationships between A1C and allcause mortality during a median 3.4-year follow-up of glycemic treatment in the ACCORD trial. Diabetes Care. 2010; 33:983-990. [PubMed: 20427682]

12. Bonds DE, Miller ME, Bergenstal RM, et al. The association between symptomatic, severe hypoglycaemia and mortality in type 2 diabetes: retrospective epidemiological analysis of the ACCORD study. BMJ. 2010; 340:b4909. [PubMed: 20061358]

13. Miller ME, Bonds DE, Gerstein HC, et al. The effects of baseline characteristics, glycaemia treatment approach, and glycated haemoglobin concentration on the risk of severe hypoglycaemia: post hoc epidemiological analysis of the ACCORD study. BMJ. 2010; 340:b5444. [PubMed: 20061360]

14. Greenfield S, Billimek J, Pellegrini F, et al. Comorbidity affects the relationship between glycemic control and cardiovascular outcomes in diabetes: a cohort study. Ann Intern Med. 2009; 151:854860. [PubMed: 20008761]

15. Holman RR, Paul SK, Bethel MA, Matthews DR, Neil HA. 10-Year follow-up of intensive glucose control in type 2 diabetes. N Engl J Med. 2008; 359:1577-1589. [PubMed: 18784090] 


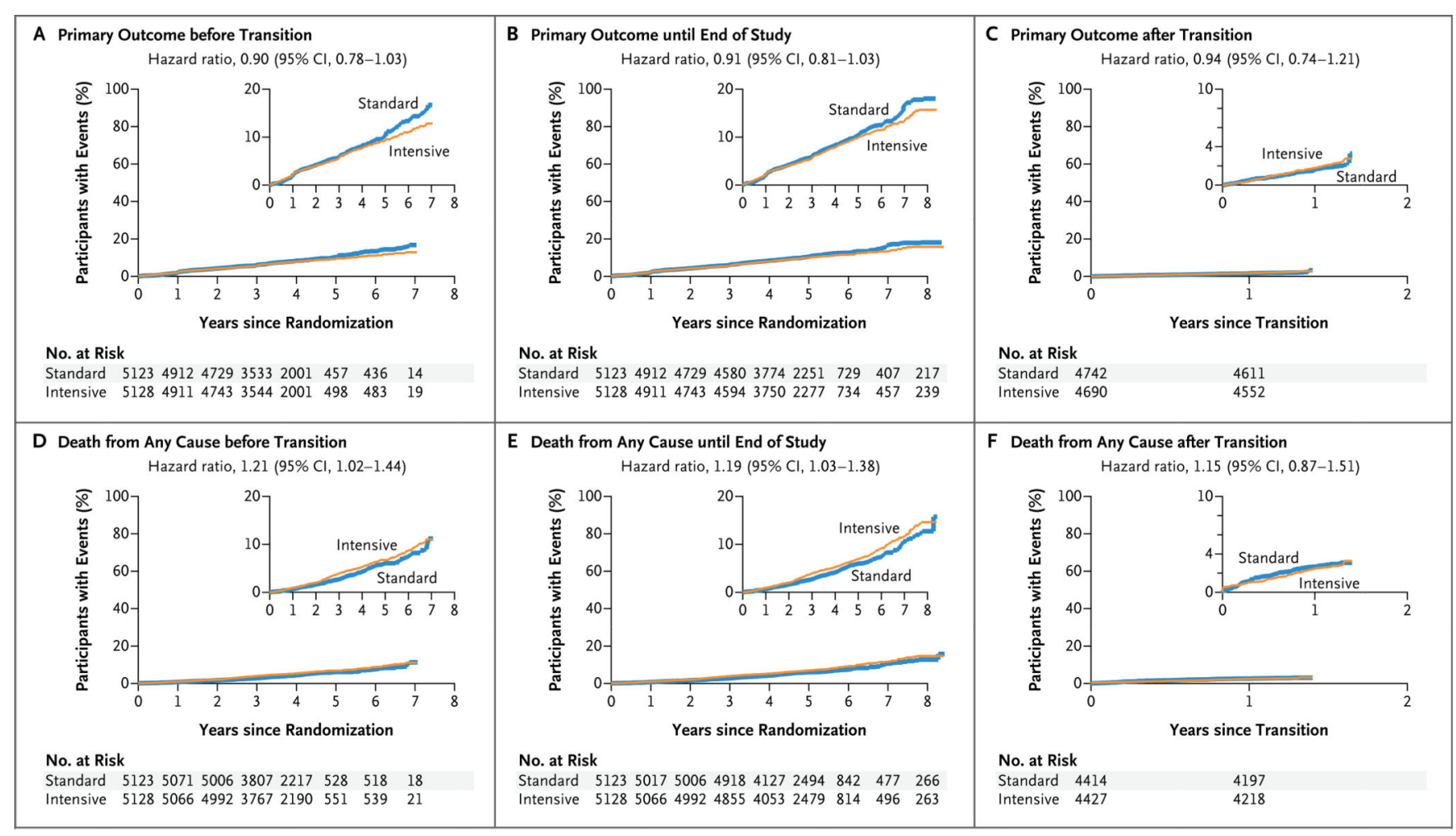

Figure 1. Kaplan-Meier Curves for the Primary Outcome and Death from Any Cause

The primary outcome was a composite of nonfatal myocardial infarction, nonfatal stroke, or death from cardiovascular causes. Panels A and D show the incidence rates from randomization until the time of transition, Panels $\mathrm{B}$ and $\mathrm{E}$ show the rates from randomization until the end of the trial, and Panels $\mathrm{C}$ and $\mathrm{F}$ show the rates for the posttransition period. Plots for the post-transition period (Panels $\mathrm{C}$ and $\mathrm{F}$ ) are included for descriptive purposes only; they cannot be used to infer any effect of the intensive therapy in this period. 


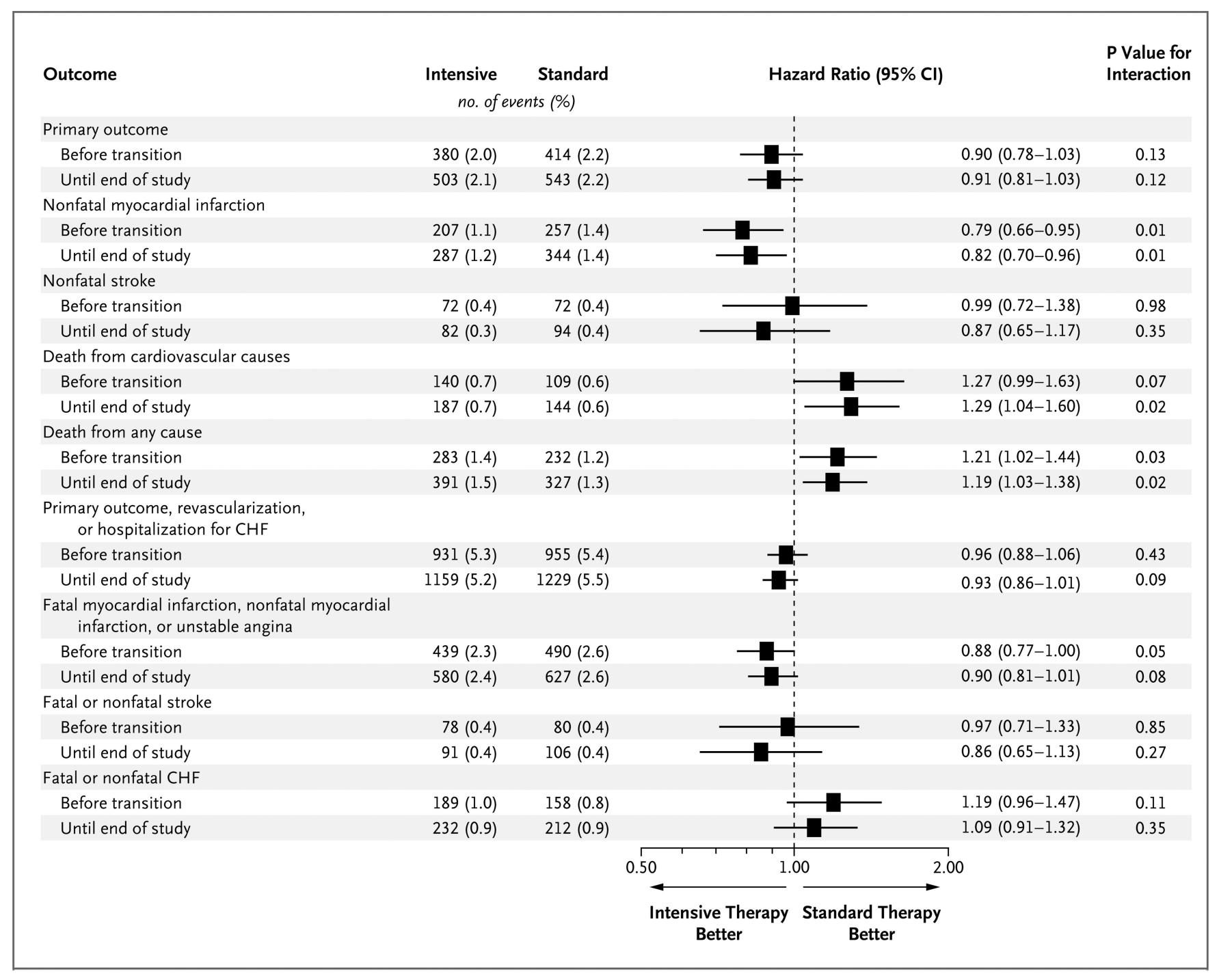

Figure 2. Hazard Ratios for the Prespecified Primary and Secondary Outcomes The effect of intensive glucose-lowering therapy is shown from randomization until the time of transition and from randomization until the end of the trial. Squares represent hazard ratios, and horizontal bars represent $95 \%$ confidence intervals. CHF denotes congestive heart failure. 


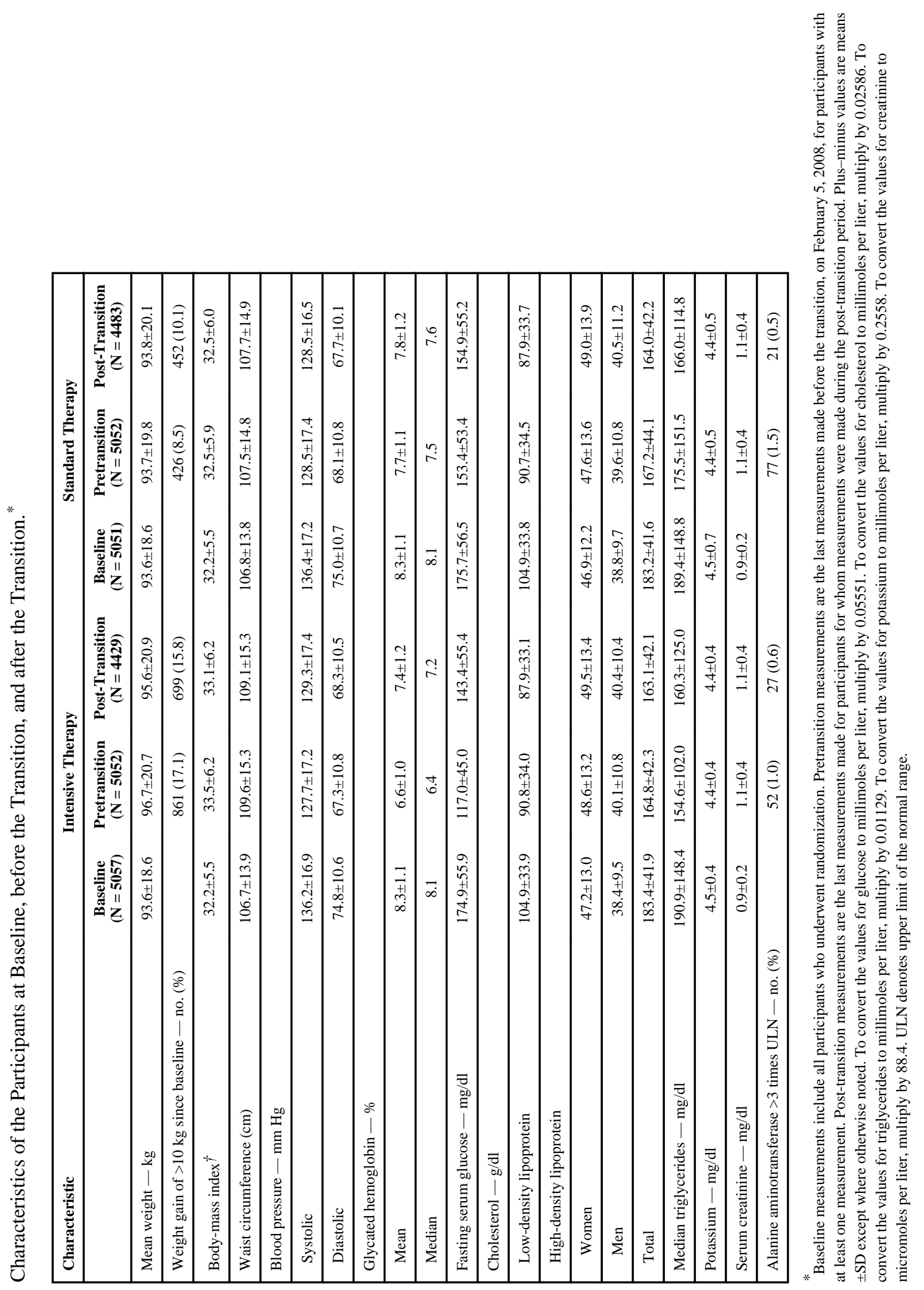

N Engl J Med. Author manuscript; available in PMC 2014 July 07. 
Page 14

N Engl J Med. Author manuscript; available in PMC 2014 July 07. 
Table 2

Causes of Death.*

\begin{tabular}{|lrrrr|}
\hline Cause of Death & \multicolumn{2}{c}{$\begin{array}{c}\text { During Pretransition } \\
\text { Period }\end{array}$} & $\begin{array}{c}\text { From Randomization } \\
\text { until End of Study }\end{array}$ \\
\hline & $\begin{array}{c}\text { Intensive } \\
\text { Therapy }\end{array}$ & $\begin{array}{c}\text { Standard } \\
\text { Therapy }\end{array}$ & $\begin{array}{c}\text { Intensive } \\
\text { Therapy }\end{array}$ & $\begin{array}{c}\text { Standard } \\
\text { Therapy }\end{array}$ \\
\hline & \multicolumn{4}{c|}{ number (percent) } \\
\hline Any & $283(5.5)$ & $232(4.5)$ & $391(7.6)$ & $327(6.4)$ \\
\hline Cardiovascular disease & & & & \\
\hline Unexpected or presumed cardiovascular disease & $89(1.7)$ & $78(1.5)$ & $124(2.4)$ & $103(2.0)$ \\
\hline Fatal myocardial infarction & $20(0.4)$ & $12(0.2)$ & $24(0.5)$ & $14(0.3)$ \\
\hline Fatal congestive heart failure & $26(0.5)$ & $20(0.4)$ & $32(0.6)$ & $25(0.5)$ \\
\hline Fatal procedure for cardiovascular disease & $11(0.2)$ & $5(0.1)$ & $14(0.3)$ & $7(0.1)$ \\
\hline Fatal arrhythmia & $4(0.1)$ & $12(0.2)$ & $6(0.1)$ & $18(0.4)$ \\
\hline Fatal procedure for noncardiovascular disease ${ }^{\dagger}$ & $1(<0.1)$ & $4(0.1)$ & $2(<0.1)$ & $4(0.1)$ \\
\hline Fatal stroke & $9(0.2)$ & $12(0.2)$ & $13(0.3)$ & $17(0.3)$ \\
\hline Other cardiovascular disease & $8(0.2)$ & $10(0.2)$ & $11(0.2)$ & $10(0.2)$ \\
\hline Cancer & $69(1.3)$ & $70(1.4)$ & $102(2.0)$ & $101(2.0)$ \\
\hline Condition other than cancer or cardiovascular disease & $57(1.1)$ & $40(0.8)$ & $84(1.6)$ & $60(1.2)$ \\
\hline Undetermined & $11(0.2)$ & $12(0.2)$ & $12(0.2)$ & $21(0.4)$ \\
\hline Identified through National Death Index & $6(0.1)$ & $1(<0.1)$ & $6(0.1)$ & $1(<0.1)$ \\
\hline
\end{tabular}

* Data within categories are not mutually exclusive, and persons who were classified as having more than one possible cause of death are listed in the relevant categories.

${ }^{\dagger}$ This condition was a component of the outcome of fatal cardiovascular disease. 


\section{Table 3}

Incident Event Rates after the Transition Date.*

\begin{tabular}{|lcccc|}
\hline Outcome & Intensive Therapy & Standard Therapy \\
\hline & $\begin{array}{c}\text { no. of } \\
\text { patients }(\%)\end{array}$ & $\begin{array}{c}\text { no. of } \\
\text { \%er year }\end{array}$ & patients (\%) & \% per year \\
\hline Primary outcome $^{\dagger}$ & $123(2.8)$ & 2.35 & $129(2.9)$ & 2.47 \\
\hline Secondary outcome & & & & \\
\hline Nonfatal myocardial infarction & $80(1.8)$ & 1.51 & $87(2.0)$ & 1.64 \\
\hline Nonfatal stroke & $10(0.2)$ & 0.18 & $22(0.5)$ & 0.40 \\
\hline Death & & & & \\
\hline Cardiovascular causes & $47(1.0)$ & 0.84 & $35(0.7)$ & 0.62 \\
\hline Any cause & $108(2.3)$ & 1.92 & $95(2.0)$ & 1.67 \\
\hline Primary outcome, revascularization, or hospitalization for heart failure & $227(5.8)$ & 4.97 & $274(7.0)$ & 6.04 \\
\hline Fatal or nonfatal myocardial infarction or unstable angina & $141(3.2)$ & 2.74 & $138(3.2)$ & 2.70 \\
\hline Fatal or nonfatal stroke & $13(0.3)$ & 0.24 & $26(0.6)$ & 0.47 \\
\hline Fatal or nonfatal congestive heart failure & $43(1.0)$ & 0.80 & $54(1.2)$ & 0.99 \\
\hline
\end{tabular}

* Data are for descriptive purposes only and cannot be used to infer any effect of the intervention during the post-transition period alone; therefore, statistical tests are not included.

${ }^{\dagger}$ The primary outcome was a composite of nonfatal myocardial infarction, nonfatal stroke, or death from cardiovascular causes. 